\title{
Correction to: Fixed Bed Hybrid Bioreactor
}

\section{Correction to:}

\section{S. Sarkar and D. Mazumder, Fixed Bed Hybrid Bioreactor,}

Green Energy and Technology, https://doi.org/10.1007/978-981-33-4546-1

The original version of the book was published without the second author. Professor Debabrata Mazumder was added as the second author retrospectively to the date of the publication and the Acknowledgements section has been updated accordingly. The correction chapter and the book have been updated. 